\title{
[3+2] Cycloaddition reaction between aliphatic alkynes and oxaziridines
}

\author{
Luigino Troisi,* Marilena Fabio, Francesca Rosato, and Valeria Videtta \\ Dipartimento di Scienze e Tecnologie Biologiche ed Ambientali, University of Salento, \\ Via Prov.le Lecce-Monteroni, 73100 Lecce, Italy \\ E-mail: luigino.troisi@unisalento.it
}

\begin{abstract}
Alkyl, aryl and acyl-substituted pyrrolidinones as an alternative to isoxazolines can be formed by [3+2] cycloaddition reactions between alkyl oxaziridines and aliphatic alkynes. The structures of isolated products change with oxaziridines Ar groups and with alkynes R and R' groups.
\end{abstract}

Keywords: Oxaziridine, isoxazoline, acyl-isoxazoline, keto-amine, pyrrolidinone

\section{Introduction}

The oxaziridines represent a class of biologically active small heterocycles ${ }^{1,2}$ whose use is becoming more prevalent in organic synthesis. The high ring strain of this three-membered ring makes it reactive towards a wide variety of substrates. For instance, it can be used both as a nitrogen transfer ${ }^{3,4}$ and oxygen transfer ${ }^{5,6}$ reagent in synthetic organic chemistry. It has also been extensively used in asymmetric synthesis. ${ }^{7,8,9,10}$ Moreover, it has been reported that oxaziridines undergo a cycloaddition reaction with a variety of heterocumulenes to afford a different set of five-membered heterocycles. ${ }^{7,8,9,10,11}$ We have recently described a simple and highly efficient [3+2] cycloaddition reaction between a variety of aryl alkenes ${ }^{12}$ or alkynes $^{13}$ and 2-alkyl-3-aryloxaziridines. This new behaviour led to 2-alkyl-3,5-diarylisoxazolidines and 2alkyl-3,5-diarylisoxazolines, respectively, as shown in Scheme 1. 


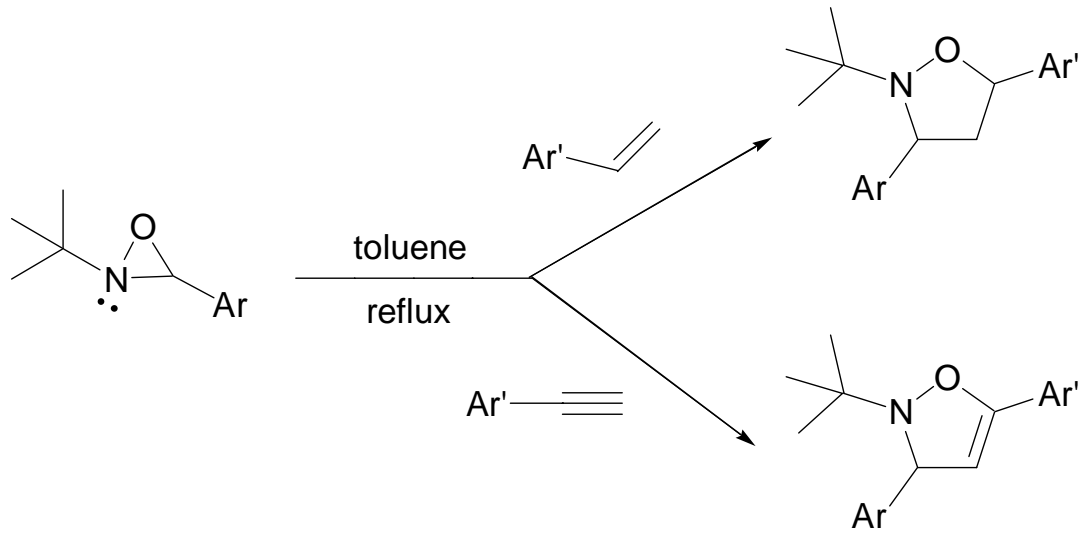

Scheme 1. [3+2] cycloaddition with terminal aryl alkenes and alkynes.

The yield of the reaction was good, with reaction time between 2 and $16 \mathrm{~h}$, depending on the Ar and Ar' type on the reactants. No product was formed with internal alkenes and alkynes such as 1-phenyl-1-propene or 1-phenyl-1-propyne, and the absence of reaction in these cases was justified as due to steric effects. Continuing our investigation, we report here the results of reactions between oxaziridines and terminal and internal aliphatic alkynes. These results are novel and unexpected, as they differ from those previously obtained.

\section{Results and Discussion}

The reaction between 3-aryl- $\mathrm{N}$-tert-butyl-oxaziridines and a terminal alkynes is represented in Scheme 2; Table 1 shows the corresponding results.

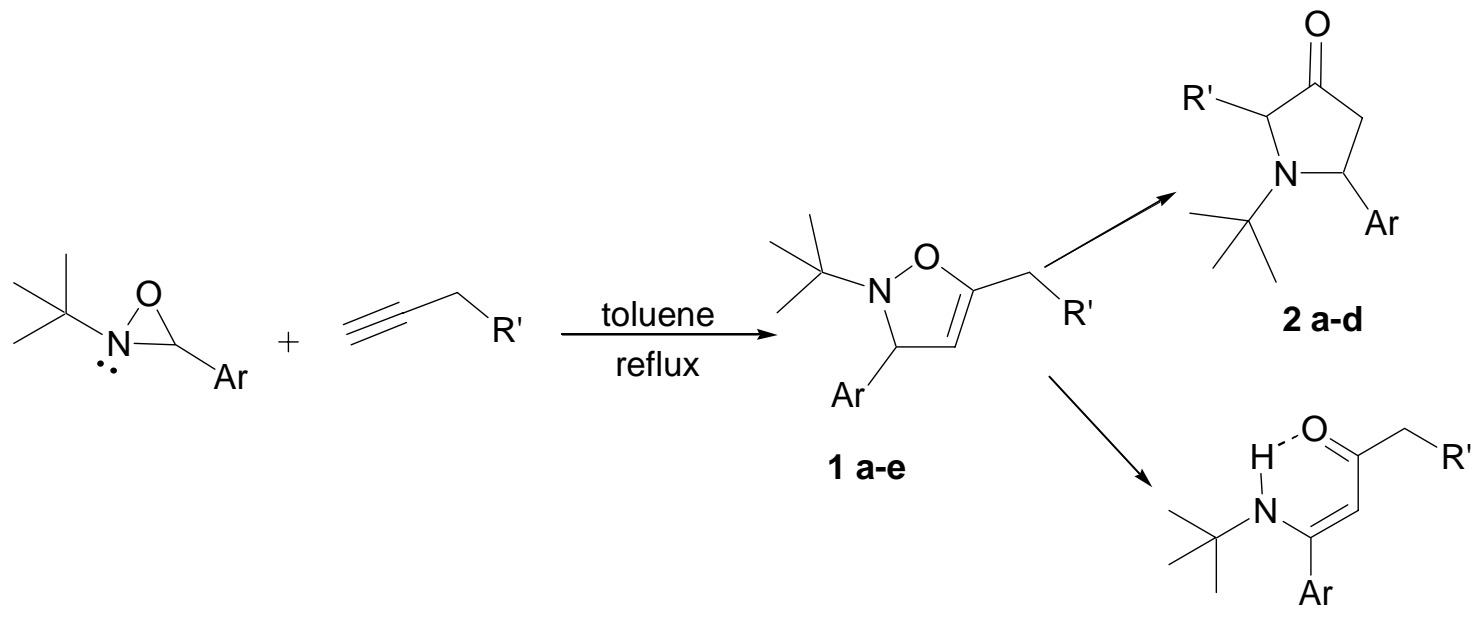

$3 a-b$

Scheme 2. $[3+2]$ cycloaddition with terminal aliphatic alkynes. 
Table 1. Reaction of oxaziridines and terminal aliphatic alkynes

\begin{tabular}{|c|c|c|c|c|c|}
\hline Entry & $\mathrm{Ar}$ & $\mathrm{R}^{\prime}$ & Time (h) & $\begin{array}{c}\text { Products distribution }{ }^{\mathrm{a}} \\
(\%)\end{array}$ & $\begin{array}{c}\text { Total yield }^{\mathrm{b}} \\
(\%)\end{array}$ \\
\hline 1 & 2(4-Me-Thiazolyl) & $n \mathrm{Pr}$ & 20 & 1a (100) & 70 \\
\hline 2 & 2-Benzothiazolyl & $n \operatorname{Pr}$ & 20 & $\mathbf{1 b}(70) 3 \mathbf{a}(30)$ & 82 \\
\hline 3 & 2-Benzothiazolyl & $n \mathrm{Pr}$ & 10 & $\mathbf{1 b}(90)$ 3a (10) & 75 \\
\hline 4 & $\mathrm{Ph}$ & $\mathrm{Br}$ & 3 & 1c $(100)$ & 91 \\
\hline 5 & 2(4-Me-Thiazolyl) & $\mathrm{Br}$ & 2 & 1d (100) & 90 \\
\hline 6 & 2-Benzothiazolyl & $\mathrm{OPh}$ & 15 & 1e (65) $3 \mathbf{b}(35)$ & 78 \\
\hline 7 & 2-Benzothiazolyl & $\mathrm{OPh}$ & 10 & $1 \mathbf{e}(80) \mathbf{3 b}(20)$ & 75 \\
\hline 8 & $\mathrm{Ph}$ & $n \operatorname{Pr}$ & 36 & 2a $(100)$ & 73 \\
\hline 9 & 4-Me-Ph & $n \mathrm{Pr}$ & 36 & $2 \mathbf{b}(100)$ & 78 \\
\hline 10 & 4-OMe-Ph & $n \operatorname{Pr}$ & 36 & 2c (100) & 71 \\
\hline 11 & 2-Pyridinyl & $n \mathrm{Pr}$ & 36 & 2d (100) & 56 \\
\hline
\end{tabular}

${ }^{a}$ Diastereomeric ratios measured by GC and ${ }^{1} \mathrm{H}$ NMR spectroscopy. ${ }^{\mathrm{b}}$ Isolated yields.

$[3+2]$ Cycloaddition reactions take place with high regioselectivity, bonding the oxygen and the nitrogen of the oxaziridine the internal and the terminal carbons of the alkyne, respectively. However different products were isolated depending on the substituents on starting materials. Isoxazoline was isolated when the starting oxaziridine carries an electron-withdrawing group on C-3, such as the thiazolyl group (entry 1, Table 1). The stable isoxazoline 1a was isolated with good yield in a relatively short reaction time, ( 20 hours). When the isoxazoline bore the benzothiazolyl group on C-3 the isoxazoline $\mathbf{1 b}$ and the keto-amine 3a were obtained in a ratio depending on the reaction time (entries $2-3$, table 1). 3a could result from the opening of $\mathbf{1} \mathbf{b}$, as a consequence of NO bond cleavage, and rearrangement with double bond shift in conjugate position. Compound 3a exist as a six-membered ring (Scheme 2) as demonstrated by the low absorption intensity showed by the carbonyl group in IR spectroscopy and by the high chemical shift value of the aminic group, NH, in ${ }^{1} \mathrm{H}$ NMR spectroscopy. Moreover, when $\mathbf{1 b}$ was refluxed in toluene, in the same reaction conditions, it was transformed in 3a in few hours, as showed by GC-MS data and ${ }^{1} \mathrm{H}$ NMR spectroscopy. Cycloaddition speed increased with terminal polarized alkynes carrying an electron-withdrawing group, such as $\mathrm{Br}$ or $\mathrm{PhO}$ in the propargylic position, entries 4-7 Table 1. Moreover, as a consequence of the presence of 2-benzothiazolyl group in 1e, the opening of isoxazoline gave compound $\mathbf{3 b}$ in a percentage depending on reaction times, (entries 6-7, Table 1). In all the other cases where 1-hexyne was used as the alkyne, 3pyrrolidinones 2 a-d (entries 8-11, Table 1) were isolated only in the cis form. The unexpected formation of pyrrolidinones is a new result. This behaviour can be justified by a double bond isomerization from internal to external side of the isoxazoline ring (A $\leftrightarrows \mathbf{B})$, Figure 1. The 
subsequent opening of $(\mathbf{B})$ to give structures $(\mathbf{C})$, showing a keto-enolic equilibrium, would be followed by the cyclization to afford the pyrrolidinone structures $\mathbf{D}$. The ${ }^{1} \mathrm{H}$ NMR spectrum of the reaction crude material showed the presence of two diastereomers for pyrrolidinone $\mathbf{D}$ (as detailed in the experimental section) in a ratio close to unity. Only the cis isomer was isolated after separation via chromatography on silica gel. In order to verify that the isomerization was generated by the silica, we monitored the reaction crude material by GC and TLC, after the addition of 2-3 $\mathrm{g}$ of silica gel. The total conversion of the trans-isomer into the cis-isomer occurred in 2-3 h. The keto-enolic equilibrium ( $\mathbf{D} \leftrightarrows \mathbf{E}$ ) and the strong steric hindrance of the tert-butyl group favour the more stable cis-isomer, Figure 1.

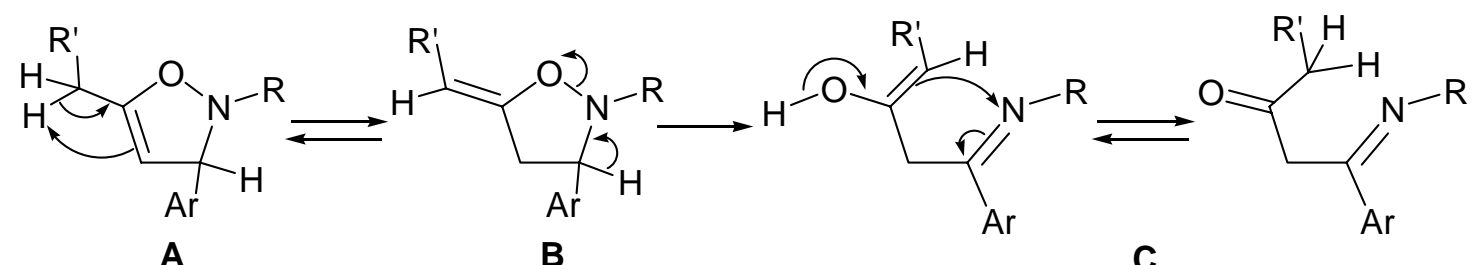

A

B

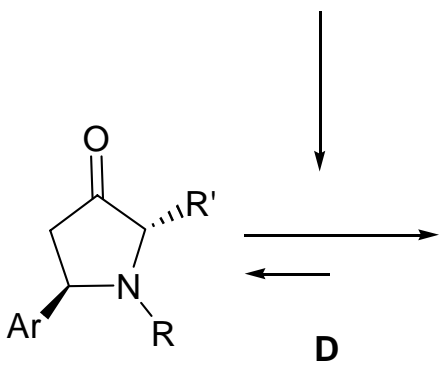<smiles>[R]C1C(=O)CC([Te])N1[R]</smiles>
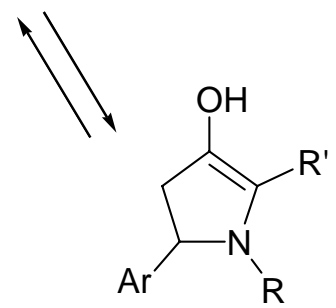

E

Figure 1. Rearrangement of isoxazolines to pyrrolidinones.

Noesy NMR experiments on the cis-3-pyrrolidinones 2 a-d showed that the tert-butyl group assumed the more stable trans configuration with respect to the other two substituents at C-2 and C-5. The reactions of the oxaziridines with internal alkynes were slower than that occurring with terminal alkynes and showed different trend and results. While the 1-phenyl-1-propyne did not give any reaction even after few days, ${ }^{7}$ an aliphatic alkyne, such as the 2- and 3-hexyne, led to 5acyl-isoxazolines with lower yields and longer reaction times $(60-90 \mathrm{~h})$, Scheme 3. 


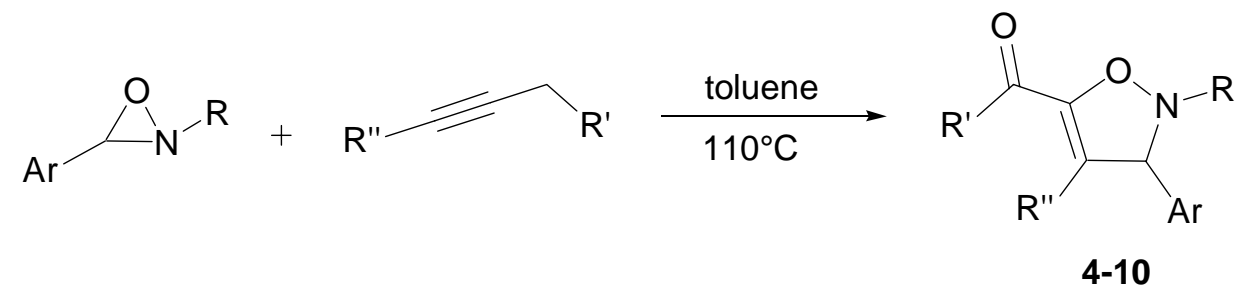

Scheme 3. [3+2] cycloaddition with internal aliphatic alkynes.

Table 2. Reaction of oxaziridines and internal aliphatic alkynes

\begin{tabular}{cccccccc}
\hline Entry & $\mathrm{Ar}$ & $\mathrm{R}$ & $\mathrm{R}^{\prime}$ & $\mathrm{R}^{\prime \prime}$ & Time (h) & Product & $\begin{array}{c}\text { Total Yield }^{\mathrm{a}} \\
(\%)\end{array}$ \\
\hline 1 & $\mathrm{Ph}$ & $t \mathrm{Bu}$ & $\mathrm{Et}$ & $\mathrm{Me}$ & 70 & $\mathbf{4}$ & 29 \\
2 & $4-\mathrm{Me}-\mathrm{Ph}$ & $t \mathrm{Bu}$ & $\mathrm{Et}$ & $\mathrm{Me}$ & 90 & $\mathbf{5}$ & 31 \\
3 & $4-\mathrm{OMe}-\mathrm{Ph}$ & $t \mathrm{Bu}$ & $\mathrm{Et}$ & $\mathrm{Me}$ & 90 & $\mathbf{6}$ & 27 \\
4 & $\mathrm{Ph}$ & $t \mathrm{Bu}$ & $\mathrm{Me}$ & $\mathrm{Et}$ & 60 & $\mathbf{7}$ & 25 \\
5 & $4-\mathrm{OMe}-\mathrm{Ph}$ & $t \mathrm{Bu}$ & $\mathrm{Me}$ & $\mathrm{Et}$ & 70 & $\mathbf{8}$ & 32 \\
6 & $\mathrm{Ph}$ & $\mathrm{Et}$ & $\mathrm{Me}$ & $\mathrm{Et}$ & 70 & $\mathbf{9}$ & 25 \\
7 & $\mathrm{Ph}$ & $i \mathrm{Pr}$ & $\mathrm{Me}$ & $\mathrm{Et}$ & 70 & $\mathbf{1 0}$ & 27 \\
\hline
\end{tabular}

${ }^{\mathrm{a}}$ Isolated yields.

We further investigate the unexpected reaction. The GC-MS analysis of the reaction mixture of entry 1, Table 2, showed the presence of traces of 4-hexyn-3-one and large amounts of benzyliden-tert-butylamine. We therefore assumed that the internal aliphatic alkyne was previously oxidized at the propargylic position by oxaziridine, forming the 4-hexyn-3-one. The carbonyl function formed in the $\alpha$ position polarizes and activates the triple bond towards the subsequent cycloaddition reaction, Scheme 4 . This trend could justify the lowest yield of this reaction calculated on the starting oxaziridine, which is partly consumed to oxidize the internal alkyne at the propargylic position. 


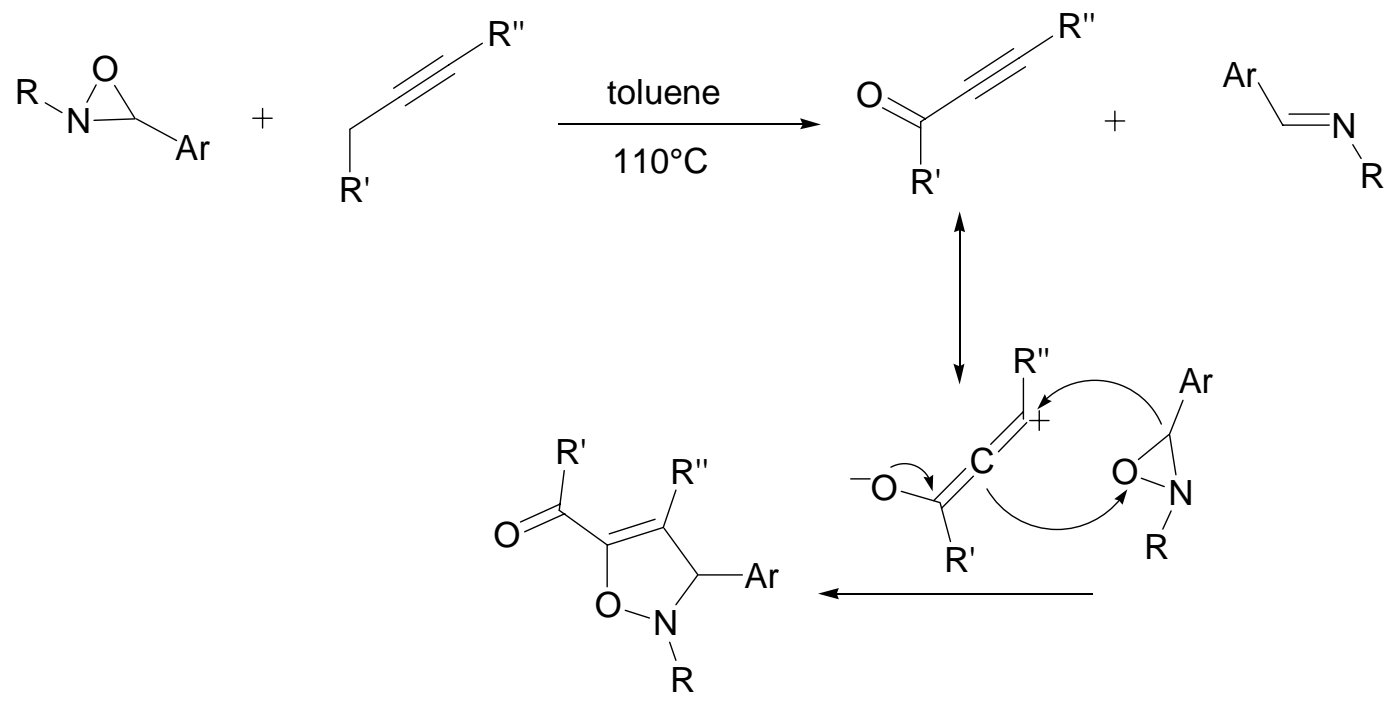

Scheme 4. Suggested mechanism of [3+2] cycloaddition with internal aliphatic alkynes.

To verify this hypothesis the reaction was carried out with internal alkynes having $\alpha$ carbonyl groups such as the 3-hexyn-2-one. This change resulted in a faster reaction (2 hours) with a higher yield (75\%), affording compound 7 as the only product.

Furthermore, when cycloaddition reaction was carried out between 4-Ph-3-butyn-2-ol and 3$\mathrm{Ph}$ - $N$-tert-butyl oxaziridine, the acyl-isoxazoline 11 was isolated as the only product (Scheme 5). Also in this case the presence of oxidized intermediate 4-Ph-3-butyn-2-one, observed in trace by ${ }^{1} \mathrm{H}$ NMR and GC-MS spectroscopy, confirmed the mechanism illustrated in Scheme 5.

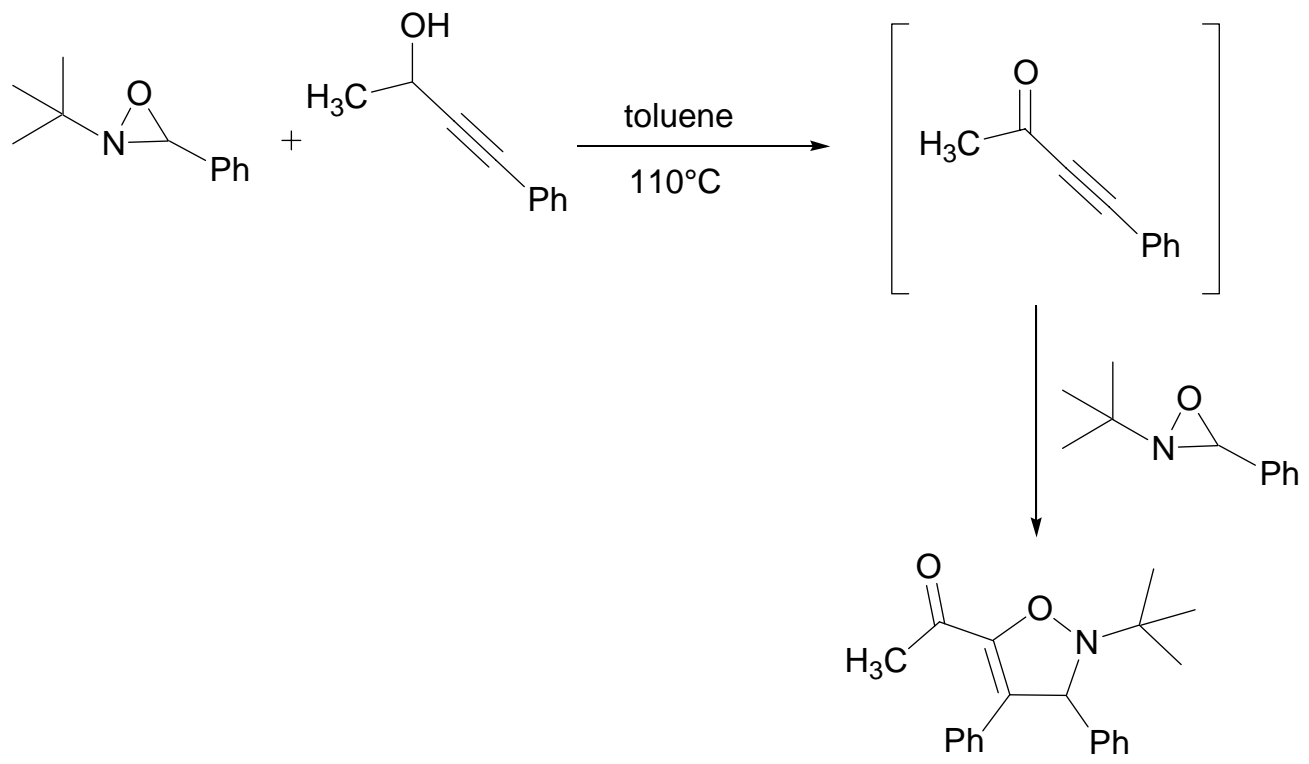

$1145 \%$ 
Scheme 5. $[3+2]$ cycloaddition with secondary propargylic alcohol.

In contrast, an internal aryl alkyne, such as 1-phenyl-1-propyne, was not able to be oxidized to a ketone, and therefore did not react.

\section{Conclusions}

In this contribution, we presented the different behaviours of oxaziridines towards the reaction with terminal and internal alkynes. The two different pathways of the [3+2] cycloaddition reaction have been proposed and justified.

Moreover, a new reactivity of oxaziridines, namely the oxidation of propargylic carbon $\mathrm{CH}_{2}$ or $\mathrm{CH}-\mathrm{OH}$ in carbonyl group, was evidenced. The possibility of obtaining alkyl, aryl, and acylsubstituted isoxazolines and pyrrolidinones by changing the starting substrates makes the presented new reactivity extremely interesting in the organic synthesis of biologically active compounds.

\section{Experimental Section}

General. All reactions were performed under an atmosphere of nitrogen in oven-dried glassware, using syringe/septum cap techniques. Reagents were generally the best quality commercial grade and used without further purification unless otherwise indicated. $\mathrm{Et}_{2} \mathrm{O}$ and $\mathrm{THF}$ were purified by distillation from sodium before use. $\mathrm{CH}_{2} \mathrm{Cl}_{2}$ was distilled from calcium hydride before use. Petroleum ether refers to the $40-60{ }^{\circ} \mathrm{C}$ boiling fraction. The imines were prepared according to Taguchi's method. ${ }^{14}$ The spectroscopic data observed were in agreement with those reported in literature for the same imines. ${ }^{12,15}$ The ${ }^{1} \mathrm{H}$ and the ${ }^{13} \mathrm{C}$ NMR spectra were recorded on a Bruker Avance 400 apparatus (400.13 and $100.62 \mathrm{MHz}$, for ${ }^{1} \mathrm{H}$ and ${ }^{13} \mathrm{C}$, respectively) with $\mathrm{CDCl}_{3}$ as solvent and TMS as internal standard $\left(\delta=7.26\right.$ for ${ }^{1} \mathrm{H}$ spectra; $\delta=77.0$ for ${ }^{13} \mathrm{C}$ spectra). The IR spectra were recorded with an FT-IR spectrophotometer Digilab Scimitar Series FTS 2000. GCMS analyses were performed with an Agilent Technologies 6850 series II gas chromatograph (5\% phenyl-polymethylsiloxane capillary column, $30 \mathrm{~m}, 0.25 \mathrm{~mm}$ i.d.), equipped with a 5973

Network mass-selective detector operating at $70 \mathrm{eV}$. The electrospray ionization (HR-ESI-MS) experiments were carried out in a hybrid QqTOF mass spectrometer (PE SCIEX-QSTAR) equipped with a ion spray ionization source. MS $(+)$ spectra were acquired by direct infusion $(5$ $\mu \mathrm{L} / \mathrm{min})$ of a solution containing the appropriate sample $(10 \mathrm{pmol} / \mu \mathrm{L})$, dissolved in a solution $0.1 \%$ acetic acid, methanol/water 50:50 at the optimum ion voltage of $4800 \mathrm{~V}$. The nitrogen gas flow was set at $30 \mathrm{psi}$ (pounds per square inch) and the potentials of the orifice, the focusing ring and the skimmer were kept at 30,50 and $25 \mathrm{~V}$ relative to ground, respectively. Melting points were determined using an Electrothermal melting point apparatus and are uncorrected. TLC was performed on Merck silica gel plates with F-254 indicator; viewing was by UV light (254 nm). 
Column chromatographies were performed on silica gel (63-200 mm) using petroleum ether/diethyl ether or petroleum ether/ethyl acetate mixtures as eluents.

\section{General procedure for the preparation of oxaziridines}

A small excess of $m$-chloroperbenzoic acid $(1.1 \mathrm{mmol})$ in $3 \mathrm{~mL}$ of $\mathrm{CH}_{2} \mathrm{Cl}_{2}$ was added to a solution of imine $(1.0 \mathrm{mmol})$ dissolved in $\mathrm{CH}_{2} \mathrm{Cl}_{2}(5 \mathrm{~mL})$ under stirring and cooling $\left(0-5{ }^{\circ} \mathrm{C}\right)$. When the reaction was complete, the excess of $m$-chlorobenzoic acid was removed by filtration. The filtrate was washed twice with a dilute solution of $\mathrm{Na}_{2} \mathrm{SO}_{3}(5 \%)$, then with a solution of $\mathrm{Na}_{2} \mathrm{CO}_{3}(5 \%)$, and finally with water. After drying over anhydrous $\mathrm{MgSO}_{4}$, the mixture was concentrated in vacuo and the crude product was purified by column chromatography (silica gel partly deactivated with triethylamine) petroleum ether : diethyl ether 95:5 for phenyl oxaziridines and petroleum ether : diethyl ether 8:2 for all other oxaziridines.

\section{General procedure for the [3+2] cycloaddition reaction}

A solution of alkyne $(1.5 \mathrm{mmol})$ and oxaziridine $(1.0 \mathrm{mmol})$ in toluene $(10 \mathrm{~mL})$ was refluxed under magnetic stirring. When TLC showed the reaction to be complete, the solution was cooled to room temperature and evaporated to dryness to give a yellow crude material. The products were isolated by flash chromatography on silica gel, petroleum ether: diethyl ether 95:5 for 1a-c, 2a, 2c, 3a, 4, 5, 8; petroleum ether: diethyl ether 90:10 for 2b, 6, 7, 9; petroleum ether: ethyl acetate 90:10 for 1d, 1e, 2d, 10; petroleum ether :diethyl ether 70:30 for $\mathbf{1 1}$ and identified by spectroscopic analysis.

5-Butyl-2-tert-butyl-3-(4-methyl-thiazol-2-yl)-2,3-dihydro-isoxazole (1a). Yield $196 \mathrm{mg}$, 70\%. Yellow oil. ${ }^{1} \mathrm{H}$ NMR (400.13 MHz, $\mathrm{CDCl}_{3}$ ): $\delta=0.89$ (t, $\left.J=7.3 \mathrm{~Hz}, 3 \mathrm{H}, \mathrm{CH}_{3} \mathrm{CH}_{2}\right), 1.15$ (s, $9 \mathrm{H}, \mathrm{C}\left(\mathrm{CH}_{3}\right)_{3}$ ), 1.35 (sextet, $J=7.3 \mathrm{~Hz}, 2 \mathrm{H}, \mathrm{CH}_{3} \mathrm{CH}_{2}$ ), 1.47 (quintet, $J=7.3 \mathrm{~Hz}, 2 \mathrm{H}$, $\left.\mathrm{CH}_{2} \mathrm{CH}_{2} \mathrm{CH}_{2}\right), 2.13\left(\mathrm{t}, J=7.3 \mathrm{~Hz}, 2 \mathrm{H}, \mathrm{CH}_{2} \mathrm{CH}_{2}\right), 2.40$ (s, 3H, $\mathrm{ArCH}$ ), 4.70 (d, $J=2.3 \mathrm{~Hz}, 1 \mathrm{H}$, $\operatorname{ArCH}), 5.42(\mathrm{~d}, J=2.3 \mathrm{~Hz}, 1 \mathrm{H}, \mathrm{C}=\mathrm{CH}), 6.78(\mathrm{~s}, 1 \mathrm{H}, \operatorname{ArH}) \mathrm{ppm} .{ }^{13} \mathrm{C}$ NMR $(100.62 \mathrm{MHz}): \delta=$ $14.1(\mathrm{q}), 17.1(\mathrm{t}), 22.3(\mathrm{t}), 24.9$ (q), 25.5 (t), 28.7 (q), 63.2 (s), 66.4 (d), 94.6 (d), 113.8 (s), 152.6 (d), 156.6 (d), 177.2 (s) ppm. FTIR $\left(\mathrm{CHCl}_{3}\right)$ : 3030 (w), 2962 (s), 2930 (s), 2874 (w), 1592 (w), 1448 (m), 1211 (vs) $\mathrm{cm}^{-1}$. GC-MS (70 eV): m/z (\%) = 280 (5) [M] $]^{+}, 265$ (15), 237 (25), 195 (12), 181 (100). HRMS (ESI): calcd. for $\mathrm{C}_{15} \mathrm{H}_{24} \mathrm{~N}_{2} \mathrm{OS}$ : (281.1609) [M+H] $]^{+}$; found: (281.1607).

5-Butyl-2-tert-butyl-3-benzohtiazol-2-yl-2,3-dihydro-isoxazole (1b). Yield $146 \mathrm{mg}, 68 \%$. Colourless oil. ${ }^{1} \mathrm{H}$ NMR (400.13 MHz, $\mathrm{CDCl}_{3}$ ): $\delta=0.90$ (t, $J=7.3 \mathrm{~Hz}, 3 \mathrm{H}, \mathrm{CH}_{3} \mathrm{CH}_{2}$ ), 1.19 (s, $9 \mathrm{H}, \mathrm{C}\left(\mathrm{CH}_{3}\right)_{3}$ ), 1.35 (sextet, $J=7.3 \mathrm{~Hz}, 2 \mathrm{H}, \mathrm{CH}_{3} \mathrm{CH}_{2}$ ), 1.51 (quintet, $J=7.3 \mathrm{~Hz}, 2 \mathrm{H}$, $\left.\mathrm{CH}_{2} \mathrm{CH}_{2} \mathrm{CH}_{2}\right), 2.17$ (t, $\left.J=7.3 \mathrm{~Hz}, 2 \mathrm{H}, \mathrm{CH}_{2} \mathrm{CH}_{2}\right), 4.76$ (d, $\left.J=1.0 \mathrm{~Hz}, 1 \mathrm{H}, \operatorname{ArCH}\right), 5.60$ (d, $J=1.0$ $\mathrm{Hz}, 1 \mathrm{H}, \mathrm{C}=\mathrm{CH}), 7.33$ (t, $J=7.9 \mathrm{~Hz}, 1 \mathrm{H}, \mathrm{ArH}), 7.43(\mathrm{t}, J=7.9 \mathrm{~Hz}, 1 \mathrm{H}, \mathrm{ArH}), 7.87(\mathrm{~d}, J=7.9 \mathrm{~Hz}$, $1 \mathrm{H}, \mathrm{ArH}), 7.94(\mathrm{~d}, J=7.9 \mathrm{~Hz}, 1 \mathrm{H}, \operatorname{ArH}) \mathrm{ppm} .{ }^{13} \mathrm{C} \operatorname{NMR}(100.62 \mathrm{MHz}): \delta=13.7$ (q), $22.3(\mathrm{t})$, 24.9 (q), 25.4 (t), 28.7 (t), 60.6 (s), 66.9 (d), 94.0 (d), 121.8 (d), 122.7 (d), 124.5 (d), 125.7 (d), 135.5 (s), 153.9 (s), 157.0 (s), 179.0 (s) ppm. FTIR (film): 3064 (w), 2960 (vs), 2931 (vs), 2871 (s), 1678 (s), 1514 (m), 1364 (s), 1211 (s), 760 (vs), 730 (s) $\mathrm{cm}^{-1}$. GC-MS (70 eV): m/z (\%) = 
316 (5) $[\mathrm{M}]^{+}, 231$ (35), 175 (100), 148 (18), 57 (10). HRMS (ESI): calcd. for $\mathrm{C}_{18} \mathrm{H}_{24} \mathrm{~N}_{2} \mathrm{OS}$ : (317.1609) $[\mathrm{M}+\mathrm{H}]^{+}$; found: (317.1611).

5-Bromomethyl-2-tert-butyl-3-phenyl-2,3-dihydro-isoxazole (1c). Yield $268 \mathrm{mg}, 91 \%$. White solid with m.p. 43-44 ${ }^{\circ} \mathrm{C}$ (n-hexane). ${ }^{1} \mathrm{H} \mathrm{NMR}\left(400.13 \mathrm{MHz}, \mathrm{CDCl}_{3}\right): \delta=1.17$ (s, 9H, $\left.\mathrm{C}\left(\mathrm{CH}_{3}\right)_{3}\right), 3.99\left(\mathrm{~s}, 2 \mathrm{H}, \mathrm{BrCH}_{2}\right), 4.98(\mathrm{~d}, J=2.4 \mathrm{~Hz}, 1 \mathrm{H}, \mathrm{ArCH}), 5.15(\mathrm{~d}, J=2.4 \mathrm{~Hz}, 1 \mathrm{H}, \mathrm{C}=\mathrm{CH})$, $7.25(\mathrm{t}, J=7.1 \mathrm{~Hz}, 1 \mathrm{H}, \operatorname{ArH}), 7.30-7.37(\mathrm{~m}, 4 \mathrm{H}, \mathrm{ArH}) \mathrm{ppm} .{ }^{13} \mathrm{C} \mathrm{NMR}(100.62 \mathrm{MHz}): \delta=21.2$ (t), 25.0 (q), 61.1 (s), 68.2 (d), 101.8 (d), 126.9 (d), 127.3 (d), 128.5 (d), 143.7 (s), 150.2 (s) ppm. FTIR $\left(\mathrm{CHCl}_{3}\right)$ : 3065 (w), 3031 (w), 2975 (vs), 2934 (w), 2873 (w), 1677 (m), 1602 (w), 1477 (m), $1366(\mathrm{~m}), 1222(\mathrm{~m}), 950(\mathrm{~m}) \mathrm{cm}^{-1}$. GC-MS compound unstable in the column chromatography. HRMS (ESI): calcd. for $\mathrm{C}_{14} \mathrm{H}_{18} \mathrm{BrNO}$ (296.0572) [M+H] $]^{+}$; found: (296.0570).

5-Bromomethyl-2-tert-butyl-3-(4-methyl-thiazol-2-yl)-2,3-dihydro-isoxazole (1d). Yield 284 mg, 90\%. Yellow oil. ${ }^{1} \mathrm{H}$ NMR (400.13 MHz, $\left.\mathrm{CDCl}_{3}\right): \delta 1.20\left(\mathrm{~s}, 9 \mathrm{H}, \mathrm{C}\left(\mathrm{CH}_{3}\right)_{3}\right), 2.41(\mathrm{~s}, 3 \mathrm{H}$, $\left.\mathrm{ArCH}_{3}\right), 3.90-3.97\left(\mathrm{~m}, 2 \mathrm{H}, \mathrm{BrCH}_{2}\right), 5.19$ (d, $\left.J=2.5 \mathrm{~Hz}, 1 \mathrm{H}, \mathrm{ArCH}\right), 5.51(\mathrm{~d}, J=2.5 \mathrm{~Hz}, 1 \mathrm{H}$, $\mathrm{C}=\mathrm{CH}), 6.82(\mathrm{~s}, 1 \mathrm{H}, \mathrm{ArH}) \mathrm{ppm} .{ }^{13} \mathrm{C} \mathrm{NMR}(100.62 \mathrm{MHz}): \delta=17.1$ (q), 20.7 (t), 24.9 (q), 61.4 (s), 66.5 (d), 114.2 (d), 128.8 (s), 151.3 (d), 152.9 (d), 175.3 (s) ppm. FTIR $\left(\mathrm{CHCl}_{3}\right)$ : 3030 (w), 2963 (w), 2928 (w), 2875 (w), 1593 (w), 1450 (m), 1210 (vs), 950 (w) cm . $^{-1}$ GC-MS compound unstable in the column chromatography. HRMS (ESI): calcd. for $\mathrm{C}_{12} \mathrm{H}_{17} \mathrm{BrN}_{2} \mathrm{OS}$ : (317.0245) $[\mathrm{M}+\mathrm{H}]^{+}$; found: (317.0246).

2-tert-Butyl-3-benzohtiazol-2-yl-5-phenoxymethyl-2,3-dihydro-isoxazole (1e). Yield $187 \mathrm{mg}$, 51\%. Yellow oil. ${ }^{1} \mathrm{H}$ NMR (400.13 MHz, $\left.\mathrm{CDCl}_{3}\right): \delta 1.26\left(\mathrm{~s}, 9 \mathrm{H}, \mathrm{C}\left(\mathrm{CH}_{3}\right)_{3}\right), 4.63(\mathrm{~s}, 2 \mathrm{H}$, $\left.\mathrm{PhOCH}_{2}\right), 5.20(\mathrm{~d}, J=2.3 \mathrm{~Hz}, 1 \mathrm{H}, \mathrm{ArCH}), 5.68(\mathrm{~d}, J=2.3 \mathrm{~Hz}, 1 \mathrm{H}, \mathrm{C}=C H), 6.90-7.00(\mathrm{~m}, 3 \mathrm{H}$, $\mathrm{PhH}), 7.18-7.26(\mathrm{~m}, 2 \mathrm{H}, \mathrm{PhH}), 7.35$ (t, $J=7.8 \mathrm{~Hz}, 1 \mathrm{H}, \mathrm{ArH}), 7.44$ (t, J=7.8 Hz, 1H, ArH), 7.87 (d, $J=7.8 \mathrm{~Hz}, 1 \mathrm{H}, \mathrm{ArH}), 7.93$ (d, $J=7.8 \mathrm{~Hz}, 1 \mathrm{H}, \mathrm{ArH}) \mathrm{ppm} .{ }^{13} \mathrm{C}$ NMR (100.62 MHz): $\delta 24.9$ (q), 61.1 (s), 66.8 (d), 71.5 (t), 98.1 (d), 115.4 (s), 121.3 (d), 121.9 (d), 122.8 (d), 124.7 (d), 125.8 (d), 128.5 (d), 129.5 (d), 135.3 (s), 143.2 (s), 152.0 (s), 177.7 (s) ppm. FTIR $\left(\mathrm{CHCl}_{3}\right): 3044$ (w), $2973(\mathrm{~m}), 2930$ (m), 1588 (s), 1491 (m), 1337 (w), $1197(\mathrm{w}) \mathrm{cm}^{-1}$. GC-MS (70 eV): m/z (\%) = $366(<1)[M]^{+}, 309$ (30), 259 (90), 203 (100), 135 (20). HRMS (ESI): calcd. for $\mathrm{C}_{21} \mathrm{H}_{22} \mathrm{~N}_{2} \mathrm{O}_{2} \mathrm{~S}$ : (367.1402) $[\mathrm{M}+\mathrm{H}]^{+}$; found: (367.1404).

(cis)-1-tert-Butyl-5-phenyl-2-propyl-pyrrolidin-3-one (2a). Yield $189 \mathrm{mg}, 73 \%$. Yellow oil. ${ }^{1} \mathrm{H}$ NMR (400.13 MHz, $\left.\mathrm{CDCl}_{3}\right): \delta=0.93\left(\mathrm{t}, J=7.0 \mathrm{~Hz}, 3 \mathrm{H}, \mathrm{CH}_{3} \mathrm{CH}_{2}\right), 1.04\left(\mathrm{~s}, 9 \mathrm{H}, \mathrm{C}\left(\mathrm{CH}_{3}\right)_{3}\right), 1_{1.34}$ $1.43\left(\mathrm{~m}, 1 \mathrm{H}, \mathrm{CH}_{2} \mathrm{CHCH}_{3}\right), 1.56-1.66\left(\mathrm{~m}, 3 \mathrm{H}, \mathrm{CH}_{2} \mathrm{CHCH}_{3}\right), 2.42(\mathrm{dd}, J=7.1,18.5 \mathrm{~Hz}, 1 \mathrm{H}$, $C H C=\mathrm{O}), 2.86(\mathrm{dd}, J=9.1,18.5 \mathrm{~Hz}, 1 \mathrm{H}, C H C=\mathrm{O}), 3.30\left(\mathrm{t}, J=6.2 \mathrm{~Hz}, 1 \mathrm{H}, \mathrm{CH}_{2} \mathrm{CHC}=\mathrm{O}\right), 4.47$ $(\mathrm{dd}, J=7.1,9.1 \mathrm{~Hz}, 1 \mathrm{H}, \mathrm{PhCH}), 7.20$ (t, $J=7.4 \mathrm{~Hz}, 1 \mathrm{H}, \mathrm{PhH}), 7.30$ (t, J=7.6 Hz, 2H, $\mathrm{PhH}$ ), 7.47 (d, $J=7.4 \mathrm{~Hz}, 2 \mathrm{H}, \mathrm{PhH}) \mathrm{ppm} .{ }^{13} \mathrm{C} \mathrm{NMR}(100.62 \mathrm{MHz}): \delta=14.2$ (q), 19.0 (t), 27.6 (q), 39.4 $(\mathrm{t}), 46.7(\mathrm{t}), 55.7(\mathrm{~s}), 57.5(\mathrm{~d}), 64.6(\mathrm{~d}), 126.2(\mathrm{~d}), 126.4$ (d), 128.3 (d), 148.4 (s), 218.2 (s) ppm. FTIR $\left(\mathrm{CHCl}_{3}\right)$ : 3029 (w), 2963 (s), 2929 (s), 1740 (s), 1466 (m), 1220 (s) cm ${ }^{-1}$. GC-MS (70 eV): $\mathrm{m} / \mathrm{z}(\%)=259(3)[\mathrm{M}]^{+}, 244(21), 216(30), 160$ (100), 104 (30), 57 (55). HRMS (ESI): calcd. for $\mathrm{C}_{17} \mathrm{H}_{25} \mathrm{NO}$ : (260.1936) [M+H] $]^{+}$; found: (260.1938).

(trans)-1-tert-Butyl-5-phenyl-2-propyl-pyrrolidin-3-one (2a). Not isolate, but seen in the crude of reaction mixture by ${ }^{1} \mathrm{H}$ NMR. On silica gel chromatography trans isomer was transformed in 
cis 2a. ${ }^{1} \mathrm{H}$ NMR $\left(400.13 \mathrm{MHz}, \mathrm{CDCl}_{3}\right): \delta 0.93\left(\mathrm{t}, J=7.0 \mathrm{~Hz}, 3 \mathrm{H}, \mathrm{CH}_{3} \mathrm{CH}_{2}\right), 1.03(\mathrm{~s}, 9 \mathrm{H}$, $\left.\mathrm{C}\left(\mathrm{CH}_{3}\right)_{3}\right), 1.34-1.43\left(\mathrm{~m}, 1 \mathrm{H}, \mathrm{CH}_{2} \mathrm{CHCH}_{3}\right), 1.56-1.66\left(\mathrm{~m}, 3 \mathrm{H}, \mathrm{CH}_{2} \mathrm{CHCH}_{3}\right), 2.26$ (dd, $J=4.0$, $17.5 \mathrm{~Hz}, 1 \mathrm{H}, C H \mathrm{C}=\mathrm{O}), 2.93(\mathrm{dd}, J=8.7,17.6 \mathrm{~Hz}, 1 \mathrm{H}, C H \mathrm{C}=\mathrm{O}), 3.75(\mathrm{dd}, J=6.1,4.0 \mathrm{~Hz}, 1 \mathrm{H}$, $\left.\mathrm{CH}_{2} \mathrm{CHC}=\mathrm{O}\right), 4.64(\mathrm{dd}, J=4.0,8.7 \mathrm{~Hz}, 1 \mathrm{H}, \mathrm{PhCH}), 7.20(\mathrm{t}, J=7.4 \mathrm{~Hz}, 1 \mathrm{H}, \mathrm{PhH}), 7.30(\mathrm{t}, J=$ $7.6 \mathrm{~Hz}, 2 \mathrm{H}, \mathrm{PhH}), 7.47$ (d, J=7.4 Hz, 2H, $\mathrm{PhH}) \mathrm{ppm}$.

(cis)-1-tert-Butyl-2-propyl-5-p-tolyl-pyrrolidin-3-one (2b). Yield $213 \mathrm{mg}, 78 \%$. Colourless oil. ${ }^{1} \mathrm{H}$ NMR $\left(400.13 \mathrm{MHz}, \mathrm{CDCl}_{3}\right): \delta=0.92\left(\mathrm{t}, J=6.8 \mathrm{~Hz}, 3 \mathrm{H}, \mathrm{CH}_{3} \mathrm{CH}_{2}\right), 1.04\left(\mathrm{~s}, 9 \mathrm{H}, \mathrm{C}\left(\mathrm{CH}_{3}\right)_{3}\right)$, 1.34-1.43 (m, 1H, $\left.\mathrm{CH}_{2} \mathrm{CHCH}_{3}\right), 1.58-1.64\left(\mathrm{~m}, 3 \mathrm{H}, \mathrm{CH}_{2} \mathrm{CHCH}_{3}\right), 2.33$ (s, 3H, $\left.\mathrm{PhCH}_{3}\right), 2.40$ (dd, $J=7.0,18.4 \mathrm{~Hz}, 1 \mathrm{H}, C H \mathrm{C}=\mathrm{O}), 2.85(\mathrm{dd}, J=9.0,18.4 \mathrm{~Hz}, 1 \mathrm{H}, C H \mathrm{C}=\mathrm{O}), 3.28(\mathrm{t}, J=6.1 \mathrm{~Hz}, 1 \mathrm{H}$, $\left.\mathrm{CH}_{2} \mathrm{CHC}=\mathrm{O}\right), 4.44(\mathrm{dd}, J=7.0,9.0 \mathrm{~Hz}, 1 \mathrm{H}, \mathrm{PhCH}), 7.12(\mathrm{~d}, J=7.8 \mathrm{~Hz}, 2 \mathrm{H}, \mathrm{PhH}), 7.35(\mathrm{~d}, J=$ $7.8 \mathrm{~Hz}, 2 \mathrm{H}, \mathrm{PhH}) \mathrm{ppm} .{ }^{13} \mathrm{C}$ NMR (100.62 MHz): $\delta 14.2$ (q), 19.0 (t), 21.0 (q), 27.6 (q), $39.4(\mathrm{t})$, $46.8(\mathrm{t}), 55.7$ (s), 57.2 (d), 64.6 (d), 126.2 (d), 129.0 (d), 135.9 (s), 145.2 (s), 218.1 (s) ppm. FTIR $\left(\mathrm{CHCl}_{3}\right)$ : 3026 (w), 2963 (s), 2930 (s), 2873 (m), 1738 (vs), 1510 (m), 1466 (m), 1200 (m) $\mathrm{cm}^{-1}$. GC-MS (70 eV): m/z (\%) = $273(4)[\mathrm{M}]^{+}, 258$ (24), 230 (32), 174 (100), 118 (31), 57 (15). HRMS (ESI): calcd. for $\mathrm{C}_{18} \mathrm{H}_{27} \mathrm{NO}$ : (274.2093) [M+H] ${ }^{+}$; found: (274.2090).

(cis)-1-tert-Butyl-5-(4-methoxy-phenyl)-2-propyl-pyrrolidin-3-one (2c). Yield $205 \mathrm{mg}$, 71\%. Colourless oil. ${ }^{1} \mathrm{H}$ NMR $\left(400.13 \mathrm{MHz}, \mathrm{CDCl}_{3}\right): \delta 0.92$ (t, $J=6.9 \mathrm{~Hz}, 3 \mathrm{H}, \mathrm{CH}_{3} \mathrm{CH}_{2}$ ), $1.04(\mathrm{~s}, 9 \mathrm{H}$, $\left.\mathrm{C}\left(\mathrm{CH}_{3}\right)_{3}\right), 1.33-1.44\left(\mathrm{~m}, 1 \mathrm{H}, \mathrm{CH}_{2} \mathrm{CHCH}_{3}\right), 1.54-1.63\left(\mathrm{~m}, 3 \mathrm{H}, \mathrm{CH}_{2} \mathrm{CHCH}_{3}\right), 2.39$ (dd, $J=7.0$, $18.7 \mathrm{~Hz}, 1 \mathrm{H}, C H \mathrm{C}=\mathrm{O}), 2.83(\mathrm{dd}, J=9.0,18.7 \mathrm{~Hz}, 1 \mathrm{H}, C H C=\mathrm{O}), 3.28(\mathrm{t}, J=6.1 \mathrm{~Hz}, 1 \mathrm{H}$, $\left.\mathrm{CH}_{2} \mathrm{CHC}=\mathrm{O}\right), 3.80\left(\mathrm{~s}, 3 \mathrm{H}, \mathrm{OCH}_{3}\right), 4.42(\mathrm{dd}, J=7.0,9.0 \mathrm{~Hz}, 1 \mathrm{H}, \mathrm{PhCH}), 6.85(\mathrm{~d}, J=8.6 \mathrm{~Hz}, 2 \mathrm{H}$, $\mathrm{PhH}), 7.37$ (d, $J=8.6 \mathrm{~Hz}, 2 \mathrm{H}, \mathrm{PhH}) \mathrm{ppm} .{ }^{13} \mathrm{C}$ NMR (100.62 MHz): $\delta 14.2(\mathrm{q}), 19.0(\mathrm{t}), 27.6(\mathrm{q})$, $39.4(\mathrm{t}), 46.8(\mathrm{t}), 55.2(\mathrm{q}), 55.7(\mathrm{~s}), 56.9(\mathrm{~d}), 64.5(\mathrm{~d}), 113.6(\mathrm{~d}), 127.2(\mathrm{~d}), 140.2(\mathrm{~s}), 158.2(\mathrm{~s})$, 217.9 (s) ppm. FTIR ( $\mathrm{CHCl}_{3}$ ): 3028 (w), 2961 (vs), 2928 (vs), 2856 (m), 1738 (vs), 1610 (m), 1509 (s), 1245 (s), $1180(\mathrm{~m}) \mathrm{cm}^{-1}$. GC-MS (70 eV): m/z (\%) = 289 (5) [M] $]^{+}, 274$ (22), 246 (41), 190 (100), 134 (71), 112 (15), 57 (18). HRMS (ESI): calcd. for $\mathrm{C}_{18} \mathrm{H}_{27} \mathrm{NO}_{2}:(290.2042)[\mathrm{M}+\mathrm{H}]^{+}$; found: (290.2044).

(cis)-1-tert-Butyl-2-propyl-5-pyridin-2-yl-pyrrolidin-3-one (2d). Yield $146 \mathrm{mg}, 56 \%$. Yellow oil. ${ }^{1} \mathrm{H}$ NMR (400.13 MHz, $\left.\mathrm{CDCl}_{3}\right): \delta=0.90\left(\mathrm{t}, J=7.0 \mathrm{~Hz}, 3 \mathrm{H}, \mathrm{CH}_{3} \mathrm{CH}_{2}\right), 1.09(\mathrm{~s}, 9 \mathrm{H}$, $\left.\mathrm{C}\left(\mathrm{CH}_{3}\right)_{3}\right), 1.35-1.44\left(\mathrm{~m}, 1 \mathrm{H}, \mathrm{CH}_{2} \mathrm{CHCH}_{3}\right), 1.50-1.61\left(\mathrm{~m}, 3 \mathrm{H}, \mathrm{CH}_{2} \mathrm{CHCH}_{3}\right), 2.67$ (dd, $J=5.8$, $18.8 \mathrm{~Hz}, 1 \mathrm{H}, C H \mathrm{C}=\mathrm{O}), 2.97(\mathrm{dd}, J=10.0,18.8 \mathrm{~Hz}, 1 \mathrm{H}, C H \mathrm{C}=\mathrm{O}), 3.34(\mathrm{t}, J=6.4 \mathrm{~Hz}, 1 \mathrm{H}$, $\left.\mathrm{CH}_{2} \mathrm{CHC}=\mathrm{O}\right), 4.63(\mathrm{dd}, J=5.8,10.0 \mathrm{~Hz}, 1 \mathrm{H}, \mathrm{ArCH}), 7.12(\mathrm{dd}, J=5.7,7.0 \mathrm{~Hz}, 1 \mathrm{H}, \mathrm{ArH}), 7.68$ $(\mathrm{t}, J=7.0 \mathrm{~Hz}, 1 \mathrm{H}, \operatorname{ArH}), 7.85(\mathrm{~d}, J=7.0 \mathrm{~Hz}, 1 \mathrm{H}, \mathrm{ArH}), 8.48(\mathrm{~d}, J=5.7 \mathrm{~Hz}, 1 \mathrm{H}, \operatorname{Ar} H) \mathrm{ppm} .{ }^{13} \mathrm{C}$ NMR (100.62 MHz): $\delta=14.1(\mathrm{q}), 19.1(\mathrm{t}), 27.4(\mathrm{q}), 38.7(\mathrm{t}), 44.2(\mathrm{t}), 55.8(\mathrm{~s}), 59.2(\mathrm{~d}), 64.5(\mathrm{~d})$, 120.9 (d), 121.5 (d), 136.5 (d), 148.6 (d), 166.7 (s), 218.3 (s) ppm. FTIR $\left(\mathrm{CHCl}_{3}\right): 3028$ (w), 2964 (vs), 2933 (s), 2874 (m), 1740 (vs), 1592 (m), 1467 (m), 1435 (m), 1369 (m), 1181 (w) cm 1. GC-MS $(70 \mathrm{eV}): m / z(\%)=260(2)[\mathrm{M}]^{+}, 245$ (28), 217 (47), 161 (100), 106 (40), 57 (18). HRMS (ESI): calcd. for $\mathrm{C}_{16} \mathrm{H}_{24} \mathrm{~N}_{2} \mathrm{O}$ : (261.1889) [M+H] $]^{+}$; found: (261.1890).

1-Benzothiazol-2-yl-1-tert-butylamino-hept-1-en-3-one (3a). Yield $79 \mathrm{mg}, 25 \%$. Colourless oil. ${ }^{1} \mathrm{H}$ NMR $\left(400.13 \mathrm{MHz}, \mathrm{CDCl}_{3}\right): \delta=0.91\left(\mathrm{t}, J=7.3 \mathrm{~Hz}, 3 \mathrm{H}, \mathrm{CH}_{3} \mathrm{CH}_{2}\right), 1.31(\mathrm{~s}, 9 \mathrm{H}$, $\left.\mathrm{C}\left(\mathrm{CH}_{3}\right)_{3}\right), 1.25-1.38\left(\mathrm{~m}, 2 \mathrm{H}, \mathrm{CH}_{3} \mathrm{CH}_{2}\right), 1.57-1.68\left(\mathrm{~m}, 2 \mathrm{H}, \mathrm{CH}_{2} \mathrm{CH}_{2} \mathrm{CH}_{2}\right), 2.35$ (t, J = 7.3 Hz, $2 \mathrm{H}$, 
$\mathrm{CH}_{2} \mathrm{CO}$ ), $5.30(\mathrm{~s}, 1 \mathrm{H}, \mathrm{CH}=\mathrm{C}), 7.49$ (t, $\left.J=8.2 \mathrm{~Hz}, 1 \mathrm{H}, \mathrm{ArH}\right), 7.55$ (t, $\left.J=8.0 \mathrm{~Hz}, 1 \mathrm{H}, \mathrm{ArH}\right), 7.93$ $(\mathrm{d}, J=8.0 \mathrm{~Hz}, 1 \mathrm{H}, \mathrm{ArH}), 8.11(\mathrm{~d}, J=8.2 \mathrm{~Hz}, 1 \mathrm{H}, \mathrm{ArH}), 11.0(\mathrm{~s}$ broad, $1 \mathrm{H}, N H) \mathrm{ppm} .{ }^{13} \mathrm{C}$ NMR $(100.62 \mathrm{MHz}): \delta=13.9(\mathrm{q}), 22.5$ (t), 27.8 (t), 31.1 (q), 42.3 (t), 54.3 (s), 99.0 (d), 121.5 (d), 124.0 (d), 126.2 (d), 126.6 (d), 135.2 (s), 152.7 (s), 154.6 (s), 163.0 (s), 199.4 (s) ppm. FTIR $\left(\mathrm{CHCl}_{3}\right): 3515$ (br w), 3067 (w), 2963 (s), 2931 (s), 2873 (w), 1588 (vs), 1478 (m), 1368 (w), $1196(\mathrm{w}), 1129(\mathrm{w}) \mathrm{cm}^{-1}$. GC-MS (70 eV): m/z (\%) = $316(20)[\mathrm{M}]^{+}, 259$ (89), 203 (100), 161 (65), 57 (25). HRMS (ESI): calcd. for $\mathrm{C}_{18} \mathrm{H}_{24} \mathrm{~N}_{2} \mathrm{OS}$ : (317.1609) [M+H] $]^{+}$; found: (317.1611).

4-Benzothiazol-2-yl-4-tert-butylamino-1-phenoxy-but-3-en-2-one (3b). Yield $99 \mathrm{mg}, 27 \%$. Yellow oil. ${ }^{1} \mathrm{H}$ NMR (400.13 MHz, $\left.\mathrm{CDCl}_{3}\right): \delta=1.35\left(\mathrm{~s}, 9 \mathrm{H}, \mathrm{C}\left(\mathrm{CH}_{3}\right)_{3}\right), 4.55\left(\mathrm{~s}, 2 \mathrm{H}, \mathrm{OCH}_{2}\right), 5.65$ (s, 1H, CH=C), 6.90-6.97 (m, 3H, PhH), 7.17-7.27 (m, 2H, PhH), 7.47 (t, J=7.9 Hz, 1H, ArH), $7.56(\mathrm{t}, J=7.9 \mathrm{~Hz}, 1 \mathrm{H}, \operatorname{ArH}), 7.93(\mathrm{~d}, J=7.9 \mathrm{~Hz}, 1 \mathrm{H}, \operatorname{ArH}), 8.10$ (d, $J=7.9 \mathrm{~Hz}, 1 \mathrm{H}, \operatorname{ArH}), 11.2$ (s broad, 1H, NH) ppm. ${ }^{13} \mathrm{C}$ NMR (100.62 MHz): $\delta=31.0$ (q), 54.9 (s), 71.5 (t), 94.8 (d), 114.7 (d), 121.2 (d), 121.6 (d), 124.1 (d), 126.4 (d), 126.7 (d), 129.5 (d), 152.6 (s), 156.5 (s), 158.2 (s), 162.3 (s), 193.0 (s) ppm. FTIR $\left(\mathrm{CHCl}_{3}\right)$ : 3500 (br w), 3043 (w), 3008 (m), 2968 (m), 2928 (m), 1590 (vs), 1484 (m), 1337 (m), 1200 (m) cm ${ }^{-1}$. GC-MS (70 eV): m/z (\%) = 366 (5) [M] $]^{+}, 309$ (18), 259 (85), 203 (100), 161 (30). HRMS (ESI): calcd. for $\mathrm{C}_{21} \mathrm{H}_{22} \mathrm{~N}_{2} \mathrm{O}_{2} \mathrm{~S}$ : (367.1402) [M+H] found: (367.1401).

1-(2-tert-Butyl-4-methyl-3-phenyl-2,3-dihydro-isoxazol-5-yl)-propan-1-one (4). Yield 79 mg, 29\%. Yellow oil. ${ }^{1} \mathrm{H}$ NMR (400.13 MHz, $\left.\mathrm{CDCl}_{3}\right): \delta=0.84$ (t, $\left.J=7.3 \mathrm{~Hz}, 3 \mathrm{H}, \mathrm{CH}_{3} \mathrm{CH}_{2}\right), 1.07$ (s, $\left.9 \mathrm{H}, \mathrm{C}\left(\mathrm{CH}_{3}\right)_{3}\right), 2.00-2.10\left(\mathrm{~m}, 1 \mathrm{H}, \mathrm{CH}_{3} \mathrm{CH}\right), 2.18-2.28\left(\mathrm{~m}, 1 \mathrm{H}, \mathrm{CH}_{3} \mathrm{CH}\right), 2.29\left(\mathrm{~s}, 3 \mathrm{H}, \mathrm{CH}_{3} \mathrm{C}=\mathrm{C}\right)$, $5.27(\mathrm{~s}, 1 \mathrm{H}, \mathrm{PhCH}), 7.15-7.27(\mathrm{~m}, 5 \mathrm{H}, \mathrm{PhH}) \mathrm{ppm} .{ }^{13} \mathrm{C} \mathrm{NMR}(100.62 \mathrm{MHz}): \delta=12.8(\mathrm{q}), 22.6$ (q), 24.9 (q), 34.5 (t), 61.2 (s), 67.9 (d), 114.0 (s), 127.5 (d), 127.7 (d), 128.5 (d), 143.4 (s), 164.0 (s), 196.1 (s) ppm. FTIR $\left(\mathrm{CHCl}_{3}\right)$ : 3032 (w), 2978 (s), 2931 (s), 1626 (m), 1600 (vs), 1456 (m), $1367(\mathrm{~m}), 1205(\mathrm{~m}) \mathrm{cm}^{-1}$. GC-MS (70 eV): m/z (\%) = 273 (15) [M] $]^{+}, 258$ (4), 244 (22), $216(65)$, 188 (100), 146 (60). HRMS (ESI): calcd. for $\mathrm{C}_{17} \mathrm{H}_{23} \mathrm{NO}_{2}$ : (274.1729) [M+H] $]^{+}$; found: (274.1731).

1-(2-tert-Butyl-4-methyl-3-p-tolyl-2,3-dihydro-isoxazol-5-yl)-propan-1-one (5). Yield 89 mg, 31\%. Yellow oil. ${ }^{1} \mathrm{H}$ NMR (400.13 MHz, $\left.\mathrm{CDCl}_{3}\right): \delta=0.92\left(\mathrm{t}, J=7.3 \mathrm{~Hz}, 3 \mathrm{H}, \mathrm{CH}_{3} \mathrm{CH}_{2}\right), 1.14$ (s, 9H, $\left.\mathrm{C}\left(\mathrm{CH}_{3}\right)_{3}\right), 2.09-2.19\left(\mathrm{~m}, 1 \mathrm{H}, \mathrm{CH}_{3} \mathrm{CH}\right), 2.24-2.35\left(\mathrm{~m}, 1 \mathrm{H}, \mathrm{CH}_{3} \mathrm{CH}\right), 2.32\left(\mathrm{~s}, 3 \mathrm{H}, \mathrm{CH}_{3} \mathrm{C}=\mathrm{C}\right)$, 2.36 (s, 3H, $\left.\mathrm{CH}_{3} \mathrm{Ph}\right), 5.31(\mathrm{~s}, 1 \mathrm{H}, \mathrm{PhCH}), 7.12$ (d, J=7.9 Hz, 2H, $\left.\mathrm{PhH}\right), 7.19$ (d, J= $7.9 \mathrm{~Hz}, 2 \mathrm{H}$, $\mathrm{PhH}$ ) ppm. ${ }^{13} \mathrm{C}$ NMR (100.62 MHz): $\delta=7.7$ (q), 12.8 (q), 21.1 (q), 24.9 (q), 34.5 (t), $61.2(\mathrm{~s})$, 67.6 (d), 113.9 (s), 127.6 (d), 129.3 (d), 137.2 (s), 140.5 (s), 164.1 (s), 196.2 (s). FTIR $\left(\mathrm{CHCl}_{3}\right)$ : 3031 (w), 2977 (vs), 2930 (vs), 1626 (m), 1601 (vs), 1455 (m), 1368 (m), 1205 (m) cm ${ }^{-1}$. GCMS (70 eV): m/z (\%) = $287(17)[\mathrm{M}]^{+}, 231$ (80), $216(20), 188$ (40), 174 (70), 132 (100). HRMS (ESI): calcd. for $\mathrm{C}_{18} \mathrm{H}_{25} \mathrm{NO}_{2}$ : (288.1885) $[\mathrm{M}+\mathrm{H}]^{+}$; found: (288.1884).

1-[2-tert-Butyl-3-(4-methoxy-phenyl)-4-methyl-2,3-dihydro-isoxazol-5-yl]-propan-1-one (6). Yield $82 \mathrm{mg}, 27 \%$. Yellow oil. ${ }^{1} \mathrm{H}$ NMR (400.13 $\left.\mathrm{MHz}, \mathrm{CDCl}_{3}\right): \delta=0.88(\mathrm{t}, J=7.2 \mathrm{~Hz}, 3 \mathrm{H}$, $\left.\mathrm{CH}_{3} \mathrm{CH}_{2}\right), 1.14\left(\mathrm{~s}, 9 \mathrm{H}, \mathrm{C}\left(\mathrm{CH}_{3}\right)_{3}\right), 2.08-2.17\left(\mathrm{~m}, 1 \mathrm{H}, \mathrm{CH}_{3} \mathrm{CH}\right), 2.26-2.34\left(\mathrm{~m}, 1 \mathrm{H}, \mathrm{CH}_{3} \mathrm{CH}\right), 2.37$ $\left(\mathrm{s}, 3 \mathrm{H}, \mathrm{CH}_{3} \mathrm{C}=\mathrm{C}\right), 3.79\left(\mathrm{~s}, 3 \mathrm{H}, \mathrm{OCH}_{3}\right), 5.31(\mathrm{~s}, 1 \mathrm{H}, \mathrm{PhCH}), 6.85(\mathrm{~d}, 2 \mathrm{H}, J=8.6 \mathrm{~Hz}, \mathrm{PhH}), 7.22$ $(\mathrm{d}, 2 \mathrm{H}, J=8.6 \mathrm{~Hz}, \mathrm{PhH}) \mathrm{ppm} .{ }^{13} \mathrm{C} \mathrm{NMR}(100.62 \mathrm{MHz}): \delta=7.7$ (q), 12.8 (q), 24.9 (q), 34.5 (t), 
55.2 (q), 61.1 (s), 67.3 (d), 114.0 (s), 128.8 (d), 135.8 (d), 159.0 (s), 163.7 (s), 164.0 (s), 196.2 (s) ppm. FTIR ( $\left.\mathrm{CHCl}_{3}\right): 3030$ (w), 2977 (vs), 2932 (vs), 1627 (m), 1600 (vs), 1453 (m), 1370 (m), $1206(\mathrm{~m}) \mathrm{cm}^{-1}$. GC-MS (70 eV): $\mathrm{m} / \mathrm{z}(\%)=303(28)[\mathrm{M}]^{+}, 247$ (80), $246(51), 232$ (30), $190(80)$, 148 (100), 121 (75). HRMS (ESI): calcd. for $\mathrm{C}_{18} \mathrm{H}_{25} \mathrm{NO}_{3}$ : (304.1834) [M+H] $]^{+}$found: (304.1836).

1-(2-tert-Butyl-4-ethyl-3-phenyl-2,3-dihydro-isoxazol-5-yl)-ethanone (7). Yield $68 \mathrm{mg}, 25 \%$. Colourless oil. ${ }^{1} \mathrm{H} \mathrm{NMR}\left(400.13 \mathrm{MHz}, \mathrm{CDCl}_{3}\right): \delta=1.16\left(\mathrm{~s}, 9 \mathrm{H}, \mathrm{C}\left(\mathrm{CH}_{3}\right)_{3}\right), 1.29(\mathrm{t}, \mathrm{J}=7.5 \mathrm{~Hz}$, $\left.3 \mathrm{H}, \mathrm{CH}_{3} \mathrm{CH}_{2}\right), 1.99\left(\mathrm{~s}, 3 \mathrm{H}, \mathrm{CH}_{3} \mathrm{C}=\mathrm{O}\right), 2.72-2.78\left(\mathrm{~m}, 1 \mathrm{H}, \mathrm{CH}_{3} \mathrm{CH}\right), 2.85-2.91\left(\mathrm{~m}, 1 \mathrm{H}, \mathrm{CH}_{3} \mathrm{CH}\right)$, $5.34(\mathrm{~s}, 1 \mathrm{H}, \mathrm{PhCH}), 7.24-7.32(\mathrm{~m}, 5 \mathrm{H}, \mathrm{PhH}) \mathrm{ppm} .{ }^{13} \mathrm{C}$ NMR $(100.62 \mathrm{MHz}): \delta=11.4(\mathrm{q}), 20.4$ (q), 24.9 (q), 29.5 (t), 61.3 (s), 68.1 (d), 113.7 (s), 127.6 (d), 127.7 (d), 128.6 (d), 143.5 (s), 169.0 (s), 192.8 (s) ppm. FTIR $\left(\mathrm{CHCl}_{3}\right): 3031$ (w), 2978 (vs), 2930 (vs), 1625 (vs), 1600 (vs), 1455 (m), $1365(\mathrm{~m}), 12068 \mathrm{~m}) \mathrm{cm}^{-1}$. GC-MS (70 eV): m/z (\%) = 273 (17) [M] $]^{+}, 217$ (100), 160 (48), 118 (78). HRMS (ESI): calcd. for $\mathrm{C}_{17} \mathrm{H}_{23} \mathrm{NO}_{2}$ : (274.1728) [M+H] $]^{+}$found: (274.1729).

1-[2-tert-Butyl-4-ethyl-3-(4-methoxy-phenyl)-2,3-dihydro-isoxazol-5-yl]-ethanone (8). Yield $97 \mathrm{mg}, 32 \%$. Yellow oil. ${ }^{1} \mathrm{H}$ NMR $\left(400.13 \mathrm{MHz}, \mathrm{CDCl}_{3}\right): \delta=1.15\left(\mathrm{~s}, 9 \mathrm{H}, \mathrm{C}\left(\mathrm{CH}_{3}\right)_{3}\right), 1.28(\mathrm{t}, \mathrm{J}=$ $\left.7.5 \mathrm{~Hz}, 3 \mathrm{H}, \mathrm{CH}_{3} \mathrm{CH}_{2}\right), 1.98\left(\mathrm{~s}, 3 \mathrm{H}, \mathrm{CH}_{3} \mathrm{C}=\mathrm{O}\right), 2.71-2.79\left(\mathrm{~m}, 1 \mathrm{H}, \mathrm{CH}_{3} \mathrm{CH}\right), 2.84-2.90(\mathrm{~m}, 1 \mathrm{H}$, $\left.\mathrm{CH}_{3} \mathrm{CH}\right), 3.78\left(\mathrm{~s}, 3 \mathrm{H}, \mathrm{OCH}_{3}\right), 5.30(\mathrm{~s}, 1 \mathrm{H}, \mathrm{PhCH}), 6.85$ (d, J=8.6 Hz, 2H, $\left.\mathrm{PhH}\right), 7.22(\mathrm{~d}, J=8.6$ $\mathrm{Hz}, 2 \mathrm{H}, \mathrm{PhH}) \mathrm{ppm} .{ }^{13} \mathrm{C}$ NMR (100.62 MHz): $\delta=11.3$ (q), 20.4 (q), 24.9 (q), 29.4 (t), 55.2 (q), 62.2 (s), 67.4 (d), 113.5 (d), 113.9 (s), 128.7 (d), 135.7 (s), 158.9 (s), 169.0 (s), 192.9 (s) ppm. FTIR $\left(\mathrm{CHCl}_{3}\right)$ : 3030 (w), 2980 (s), 2932 (s), 1625 (m), 1600 (vs), 1457 (m), 1360 (m), 1207 (m) $\mathrm{cm}^{-1}$. GC-MS (70 eV): m/z (\%) = 303 (20) [M] $]^{+}, 247$ (40), 246 (35), 218 (25), 204 (100). HRMS (ESI): calcd. for $\mathrm{C}_{18} \mathrm{H}_{25} \mathrm{NO}_{3}$ : (304.1834) $[\mathrm{M}+\mathrm{H}]^{+}$; found: (304.1833).

1-(2,4-Diethyl-3-phenyl-2,3-dihydro-isoxazol-5-yl)-ethanone (9). Yield $61 \mathrm{mg}, 25 \%$. Yellow oil. ${ }^{1} \mathrm{H}$ NMR (400.13 MHz, $\left.\mathrm{CDCl}_{3}\right): \delta=1.23\left(\mathrm{t}, J=7.1 \mathrm{~Hz}, 3 \mathrm{H}, \mathrm{CH}_{3} \mathrm{CH}_{2}\right.$ ), 1.27 (t, $J=7.3 \mathrm{~Hz}$, $\left.3 \mathrm{H}, \mathrm{CH}_{3} \mathrm{CH}_{2}\right), 2.00\left(\mathrm{~s}, 3 \mathrm{H}, \mathrm{CH}_{3} \mathrm{C}=\mathrm{O}\right), 2.70-2.86\left(\mathrm{~m}, 2 \mathrm{H}, \mathrm{CH}_{3} \mathrm{CH}_{2} \mathrm{C}=\mathrm{C}\right), 2.92-3.00(\mathrm{~m}, 1 \mathrm{H}$, $\left.\mathrm{CH}_{3} \mathrm{CHN}\right), 3.10-3.17\left(\mathrm{~m}, 1 \mathrm{H}, \mathrm{CH}_{3} \mathrm{CHN}\right), 5.04(\mathrm{~s}, 1 \mathrm{H}, \mathrm{PhCH}), 7.26-7.36(\mathrm{~m}, 5 \mathrm{H}, \mathrm{PhH}) \mathrm{ppm} .{ }^{13} \mathrm{C}$ NMR (100.62 MHz): $\delta=11.4$ (q), 12.0 (q), 20.5 (q), 29.7 (t), 54.5 (t), 74.3 (d), 113.7 (s), 127.4 (d), 128.0 (d), 128.7 (d), 141.5 (s), 169.0 (s), 193.2 (s) ppm. FTIR $\left(\mathrm{CHCl}_{3}\right): 3030$ (w), 2977 (vs), 2932 (vs), 1624 (vs), 1599 (m), 1454 (m), 1367 (m), 1204 (m) cm $\mathrm{cm}^{-1}$ GC-MS (70 eV): m/z (\%) = 245 (60) [M] $]^{+}, 188$ (42), 168 (72), 146 (100), 118 (70). HRMS (ESI): calcd. for $\mathrm{C}_{15} \mathrm{H}_{19} \mathrm{NO}_{2}$ : (246.1416) $[\mathrm{M}+\mathrm{H}]^{+}$; found: (246.1414).

1-(4-Ethyl-2-isopropyl-3-phenyl-2,3-dihydro-isoxazol-5-yl)-ethanone (10). Yield $70 \mathrm{mg}$, 27\%. Colourless oil. ${ }^{1} \mathrm{H} \mathrm{NMR}\left(400.13 \mathrm{MHz}, \mathrm{CDCl}_{3}\right.$ ): $\delta=0.87$ (t, $J=7.2 \mathrm{~Hz}, 3 \mathrm{H}, \mathrm{CH}_{3} \mathrm{CH}_{2}$ ); 1.25 $\left(\mathrm{d}, J=7.0 \mathrm{~Hz}, 6 \mathrm{H}, \mathrm{CH}\left(\mathrm{CH}_{3}\right)_{2}\right) ; 2.05\left(\mathrm{~s}, 3 \mathrm{H}, \mathrm{CH}_{3} \mathrm{C}=\mathrm{O}\right), 2.27$ (eptet, $\left.J=7.0 \mathrm{~Hz}, 1 \mathrm{H}, \mathrm{CH}\left(\mathrm{CH}_{3}\right)_{2}\right)$, 4.12 (q, $\left.J=7.2 \mathrm{~Hz}, 2 \mathrm{H}, \mathrm{CH}_{3} \mathrm{CH}_{2}\right), 5.01(\mathrm{~s}, 1 \mathrm{H}, \mathrm{PhCH}), 7.36-7.40(\mathrm{~m}, 5 \mathrm{H}, \mathrm{PhH}) \mathrm{ppm} .{ }^{13} \mathrm{C} \mathrm{NMR}$ (100.62 MHz): $\delta=14.2$ (q), 22.7 (q), 29.3 (q), 31.9 (t), 60.4 (d), 79.2 (d), 115.1 (s), 128.6 (d), 128.9 (d), 129.4 (d), 135.8 (s), 171.2 (s), 193.0 (s) ppm. FTIR $\left(\mathrm{CHCl}_{3}\right): 3033$ (w), 2980 (s), 2931

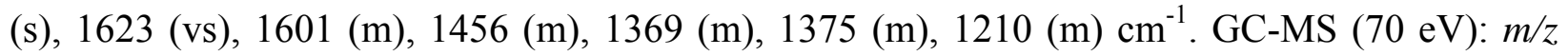
$(\%)=259(60)[\mathrm{M}]^{+}, 160$ (72), 118 (100), 105 (72), 91 (73). HRMS (ESI): calcd. for $\mathrm{C}_{16} \mathrm{H}_{21} \mathrm{NO}_{2}$ : (260.1572) $[\mathrm{M}+\mathrm{H}]^{+}$; found: (260.1574). 
1-(2-tert-Butyl-3,4-diphenyl-2,3-dihydro-isoxazol-5-yl)-ethanone (11). Yield $144 \mathrm{mg}$, 45\%. Colourless oil. ${ }^{1} \mathrm{H}$ NMR (400.13 MHz, $\left.\mathrm{CDCl}_{3}\right): \delta=1.25\left(\mathrm{~s}, 9 \mathrm{H}, \mathrm{C}\left(\mathrm{CH}_{3}\right)_{3}\right), 1.83(\mathrm{~s}, 3 \mathrm{H}$, $\left.\mathrm{CH}_{3} \mathrm{C}=\mathrm{O}\right), 5.59(\mathrm{~s}, 1 \mathrm{H}, \mathrm{PhCH}), 7.34(\mathrm{t}, J=7.4 \mathrm{~Hz}, 3 \mathrm{H}, \mathrm{PhH}), 7.46-7.54(\mathrm{~m}, 5 \mathrm{H}, \mathrm{PhH}), 7.61(\mathrm{~d}, J$ $=7.4 \mathrm{~Hz}, 2 \mathrm{H}, \mathrm{PhH}) \mathrm{ppm} .{ }^{13} \mathrm{C}$ NMR $(100.62 \mathrm{MHz}): \delta=25.0$ (q), 29.7 (q), 61.6 (s), $68.6(\mathrm{~d})$, 116.0 (s), 127.4 (s), 127.5 (d), 128.3 (d), 128.4 (d), 128.6 (d), 128.9 (s), 129.5 (d), 131.1 (d), 163.1 (s), 192.4 (s) ppm. FTIR $\left(\mathrm{CHCl}_{3}\right)$ : 3030 (w), 2927 (s), 2855 (s), 1718 (m), 1630 (s), 1453 (m), $1368(\mathrm{~m}), 1261(\mathrm{~m}) \mathrm{cm}^{-1}$. GC-MS (70 eV): m/z (\%) = 321 (12) [M] $]^{+}, 365$ (81), 364 (28), 161 (53), 105 (100), 77 (55). HRMS (ESI): calcd. for $\mathrm{C}_{21} \mathrm{H}_{23} \mathrm{NO}_{2}$ : (260.1729) [M+H] $]^{+}$; found: (260.1731).

\section{Acknowledgements}

We thank the MIUR, the University of Salento and the C.I.N.M.P.I.S. (Consorzio Interuniversitario Nazionale Metodologie e Processi Innovativi di Sintesi) for the financial support.

\section{References}

1. Gonzales, C. R.; Balogh-Nair, V. Int. J. Mol. SCI 2002, 3, 1145.

2. Hata, Y.; Watanabe, M. J. Org. Chem. 1981, 46, 610.

3. Andreae, S.; Schmitz, E. Synthesis 1991, 5, 327.

4. Armstrong, A.; Atkin, M. A.; Swallow, S. Tetrahedron Lett. 2000, 41, 2247.

5. Bohe, L.; Lusinchi, X. Tetrahedron 1999, 55, 155.

6. Jenning, W. B.; O'Shea, J. H.; Schweppe, A. Tetrahedron Lett. 2001, 42, 101.

7. Davis, F. A.; Sheppard, A. C. Tetrahedron 1989, 45, 5703.

8. Aube, J. J. Chem. Soc. Rev 1997, 26, 269.

9. Davis, F. A.; Chen, B. C. Chem. Rev. 1992, 92, 919.

10. Troisi, L.; De Lorenzis, S.; Fabio, M. ; Rosato, F.; Granito, C. Tetrahedron: Asymmetry 2008, 19, 2246.

11. Kraiem, J.; Grosvalet, L.; Perrin, M.; Hassine, B. B. Tetrahedron Lett. 2001, 42, 9131.

12. Fabio, M.; Ronzini, L.; Troisi, L. Tetrahedron 2007, 63, 12896.

13. Fabio, M.; Ronzini, L.; Troisi, L. Tetrahedron 2008, 64, 4979.

14. Taguchi, K.; Westheimer, F. H, J. Org. Chem. 1971, 36, 1570.

15. Campbell, K. N.; Helbing, C. H.; Florkowski, M. P.; Campbell, B. K. J. Am. Chem. Soc. 1948, $70,3868$. 